\title{
Measuring Device of Shallow Wave's Velocity Based on MATLAB Image Grayscale Processing \\ Dongsheng Zhang
}

North China Electric Power University, Beijing, 102206

alanncepu@foxmail.com

Keywords: Shallow wave; Grayscale processing; Pixel; Crests; Valleys

\begin{abstract}
In order to measure the shallow wave velocity and improve the accuracy of traditional observation by naked eyes, we design the measuring device of shallow wave velocity. We use dripping to start the shallow wave, capture the image at a fixed time interval for the video of shallow wave, and get grayscale processing. According to the grid images with grayscale quantitative value, the place of crest and trough can be located and then we can get the shallow waves velocity. After application test and literature verification, the device is not only easy to operate and construct but also accurate to measure. Besides, this method of measure can be applied to the task about the image processing, which is difficult for naked eye to observe.
\end{abstract}

\section{Introduction}

The measure of shallow wave velocity is a physics experiment with important research significance. As for the method to measure of shallow wave velocity now, it is demanding and baroque for the apparatus. However, the cheap apparatus always can't achieve the demand of accuracy.

MATLAB has the abilities to handle images. The grayscale processing is one of them, which provides prerequisites for measure of shallow wave velocity. Based on grayscale processing, this paper puts forward the method to determine the location of crest and trough, construct the measure device of shallow wave velocity. Besides, we conduct the experiment under some different conditions and analysis the error. The experimental results are in agreement with the theory, which validate the feasibility and accuracy.

\section{Instruments and Principles}

General steps are following:

1. Drip to start the shallow wave

2. Record the diffusion processing of the shallow wave.

3. Capture the image at a fixed time interval for the video of shallow wave.

4. Grayscale processing.

5. Determine the moving distance by grid images with grayscale quantitative value.

6. Calculate the velocity by formula at that time.

The device is shown in the fig. 1 : 


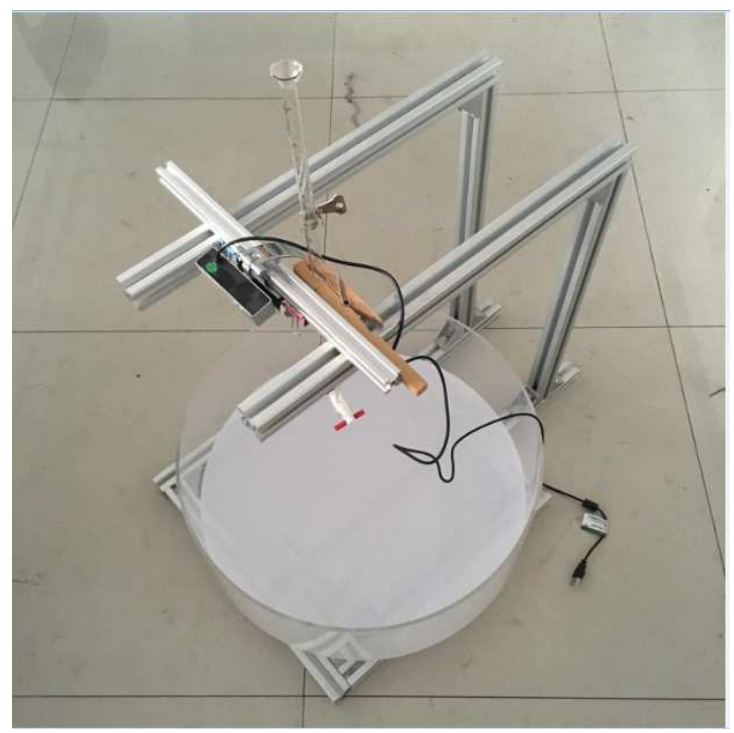

Figure 1. The device

Capture. Record the diffusion processing of the shallow wave:

1. Start the light.

2. Rotate the rotary knob of burette. When the shallow wave is stable, open the camera to video.

3. Rotate the rotary knob of burette back. Choose the video that has obvious shallow wave to get next dispose.

To dispose the video, we use the thought that we call "static state". We use software to capture the image .As we know from the pre-experiment, it is convenient to determine the location of crest and trough when the fixed time interval is $1 / 30$ second. Because the difference between two images is small, it is beneficial to follow-up processing. There are several diffusion processing images of the shallow wave after filter.

Grayscale Processing. Any color is formed by red, green and blue, which are three primary colors. For example, the original color of a point is $\operatorname{RGB}(\mathrm{R}, \mathrm{G}, \mathrm{B})$, we can use floating - point algorithm in commonly used gray processing algorithm:

Gray $=R * 0.3+G * 0.59+B * 0.1$

Let the original $\mathrm{RGB}(\mathrm{R}, \mathrm{G}, \mathrm{B})$ replaced by Gray. So we can get a new color RGB (Gray. Gray, Gray), which is called grayscale processing.

Under fixed light intensity, the crest has a condenser effect of a convex lens. It can produce a bright light stripe. The trough has opposite effect. It can produce dark stripe. There are stripes that have significant brightness difference in the image. There are difference grayscale values in the image after grayscale processing.
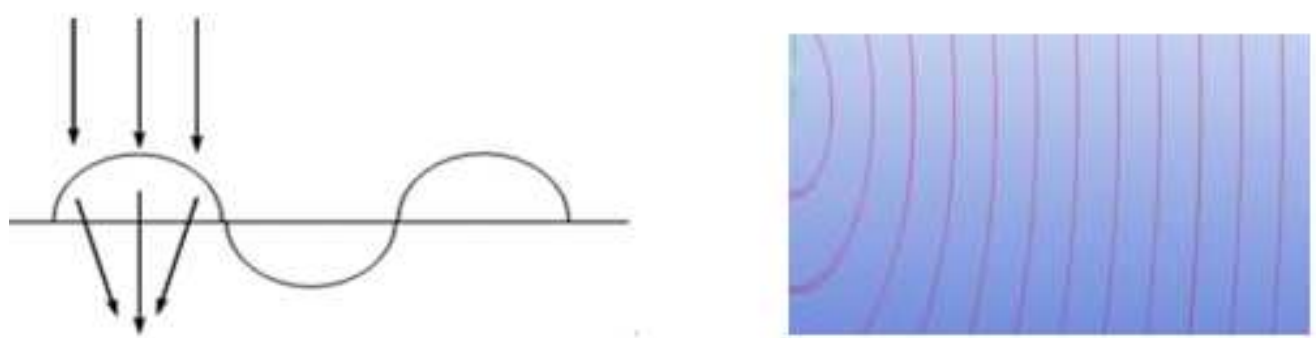

Figure 2. The shallow wave theory and simplified effect

The image can be enlarged to pixel by the function in MATLAB. It is convenient and clear to determine fine location. 
Using the image processing functions mentioned earlier, we establish X-Y coordinate system and do grayscale processing. Processed image is shown as follows:

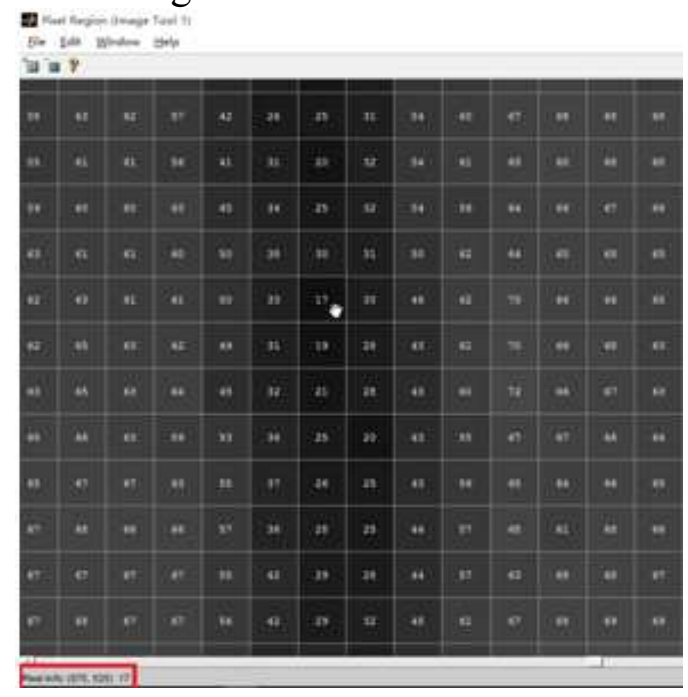

Figure 3. Image after gridded and grayscale processed

As we can see, the image is divided by fixed amount small grids. Every small grid shows the grayscale value and $\mathrm{X}-\mathrm{Y}$ coordinate value. According to the definition of grayscale, the grayscale value is higher, the point is lighter. According to a lot of experiments, the darkest point's grayscale value is 15 . As a result, we choose area whose grayscale value is 15 around to determine the location of dark stripe.

We choose three images that time interval is $1 / 30$ second. We fix the $\mathrm{Y}$ coordinate value and determine the direction of moving. According to the difference between them, we can get the distance of shallow wave moving.

Data Processing and Error Analysis. In the pre-experiment, we use ruler to be reference substance. Then we know every grid is 0.05 centimeter. From the last step, we determine the distance of shallow wave moving. To reduce the error, we choose the mean to calculate. Using the formula:

$$
V=S / T
$$

We can get the velocity. $T$ is the fix time interval.

Because the biggest error is one grid, the final result is

$$
\mathrm{V}=\left(\mathrm{V}^{\prime} \pm 0.5\right) \mathrm{mm} / \mathrm{s}
$$

\section{Extended}

We can apply this method to the university physics experiment- the experiment of equal thickness of light and improve it.

In this experiment, we need count the interference figures which are bright and dark. Because the interference figures are numerous and intensive, it is easy to make mistakes. We can determine the location of interference figure. The steps are following:

1. Connect the microscopes and camera.

2. Grayscale processing.

3. Using the grayscale value of definite location to draw a curve.

4. Using the curve to count the number of stripes.

\section{Conclusion}

From the application analysis, the measuring device of shallow wave velocity based on MATLAB can finish the measuring task accurately. The result is identical to reference. It has high precision, 
easy operation. Its cost is cheap. Besides, it is convenient to discern the number. Using the software to capture the image can make the problem easier. The grayscale processing can fix the problem that the error is big, the device is complex and it is difficult to discern the number.

\section{References}

[1] LUO Daobin, SHEN Zhirong. Application of Ultrasonic Grating Method to Measurement of Wave Velocity of Liquid Surface [J]. College Physics, 2009, 28 (2): 41-43.

[2] TONG Peixiong, ZHAO Zaizhong. Construction of vibration frequency and wave propagation velocity of water [J]. Physics Experiment, 2005, 25 (5): 6-9.

[3] Ashikhmin M. 2001 .Synthesizing natural textures. 2001 ACM Symposium on Interactive 3D Graphics (March), 217-226.

[4] PENG Chuanxi, ZHAO Guanxian, WANG Zhi-xian. Study on Gray Scale Algorithm and Efficiency Analysis Based on Image Recognition System [J]. Electronic World, 2014 (7): 105-105.

[5] LIU Yong. Study and implementation of algorithm for color gray image [D]. Shandong University, 2007.

[6] LU Yigang, TONG Jie. Study on pressure coefficient and temperature coefficient of liquid molecular free process [J]. Journal of Shaanxi Normal University (Special Edition), 1996 (2): 31-35.

[7] Huggins E. Introduction to Fourier Optics [J]. Physics Teacher, 2007, 22(45):364-368.

[8] Hong L, Jain A. Fingerprint Enhancement [M]// Automatic Fingerprint Recognition Systems. Springer New York, 2004:127-143.

[9] Kamei T, Mizoguchi M. Image filter design for fingerprint enhancement [M]// Automatic Fingerprint Recognition Systems. Springer New York, 2004:113-126.

[10] Soczhiewicz E. The dependence on pressure of the Velocity of ultrasonic waves in liquids and the available volume of mix-tures. Akchives of Acoustics, 1977,4(2): 325 329

[11]Reinhard E, Ashikhmin M, Gooch B and Shirley P. Color Transfer between Images, IEEE Computer Graphics and Applications, September/October 2001, 34-40. 